\title{
A Generative Model of Human Hair for Hair Sketching
}

\author{
Hong Chen and Song Chun Zhu \\ Departments of Statistics and Computer Science \\ University of California, Los Angeles \\ \{hchen,sczhu\}@stat.ucla.edu
}

\begin{abstract}
Human hair is a very complex visual pattern whose representation is rarely studied in the vision literature despite its important role in human recognition. In this paper, we propose a generative model for hair representation and hair sketching, which is far more compact than the physically based models in graphics. We decompose a color hair image into three bands: a color band (a) (by Luv transform), a low frequency band (b) for lighting variations, and a high frequency band (c) for the hair pattern. Then we propose a three level generative model for the hair image (c). In this model, image (c) is generated by a vector field (d) that represents hair orientation, gradient strength, and directions; and this vector field is in turn generated by a hair sketch layer (e). We identify five types of primitives for the hair sketch each specifying the orientations of the vector field on the two sides of the sketch. With the five-layer representation (a-e) computed, we can reconstruct vivid hair images and generate hair sketches. We test our algorithm on a large data set of hairs and some results are reported in the experiments.
\end{abstract}

\section{Introduction}

Human hair is a very complex visual pattern with hundreds of thousands of hairs grouped into strands and wisps which create diverse hair styles. Hair appearance plays an important role in graphics, digital human animation, as well as in human recognition. For example, it is evident that hair style has significant contributes to face recognition. If we keep the hair image of a person while replacing his/her mouth and/or nose, there is a good chance that the image is identified as the original person by people who are familiar with him/her. Therefore modeling human hair is of great interest for both graphics and vision.

In graphics, hair acquisition, modeling and animation have attracted increasing attention in recent years[8, 9, 3]. Graphics work adopted very tedious 3D hair representa-

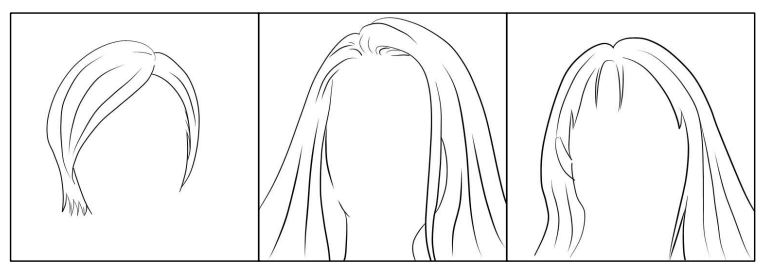

Figure 1. Three examples of artist hair drawings. The sketches are concise and grasp the essences of hair perception.

tions. For example Change et al [3] used 50,000 hair strands with physics models for the hair dynamics and friction, and Lee et al[9] used 20,000 hair strands. Most of the graphics work provided user interfaces for editing the hair interactively.

In vision, hair is usually studied as an oriented patterns [7, 12] among other textures, such as wood grain, flows, finger prints and so on. Recently few works consider modeling hair from images, such as [16]. They capture dense orientation field from images. However, there is, no specific model dedicated to understand the global hair structure.

In this paper, we propose a compact generative model for hair representation and hair sketching. We argue that it is infeasible to infer the $O\left(10^{4}\right)$ hair strands in the graphics models from a single image where the number of pixels for hair may be smaller than the number of hairs or hair strands. For the lack of information, human perception cannot perceive or may not care about many details, as it was demonstrated in texture perception[1]. Fig.1 shows three examples of artist's sketches of human hairs which grasp the essences of the hairs by concise strokes (curves). With attributes added, those curves and strokes are capable of reconstructing hair images by texture fill-in method, and the reconstructed image may appear equivalent perceptually to the original one. We test our models and algorithms in a data set of 300 human hairs extracted from a cosmetic makeup software. Some of our results are shown in Fig.9.

Our model and algorithm are illustrated in Fig.2. This 


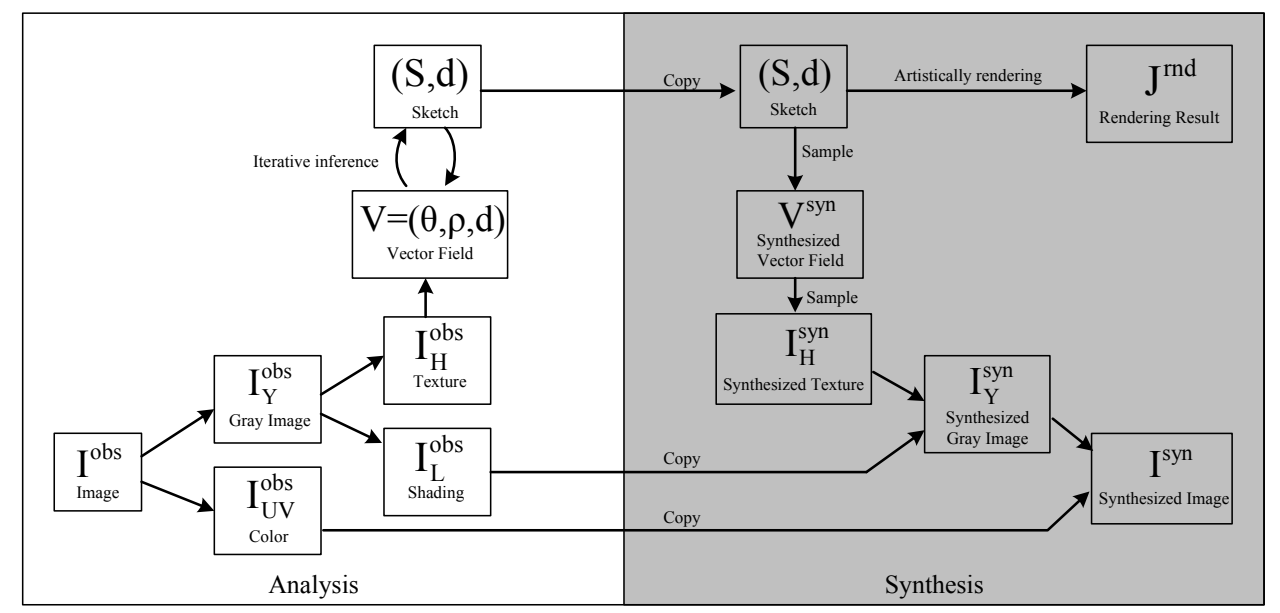

Figure 2. Flow of our algorithm. For input image $I^{\text {obs }}$, we decouple it into gray image $I_{Y}$ and color channel $I_{U V}$. The gray image further decomposed into texture part $I_{H}$ and shading $I_{L}$. Given $I_{H}$, we use iterative sketch inference algorithm to get the sketch $\mathrm{S}$ with direction $\mathrm{d}$ and the vector field $\mathrm{V}$. From the sketch result, we synthesize vector field $\mathrm{V}^{\text {syn }}$, based on which we can synthesize the hair image $I_{H}^{\text {syn }}$. The latter is combined with the shading $I_{L}$ and color $I_{U V}$, and yields the final synthesized result $\mathbf{I}^{\text {syn }}$. We can render the cartoon sketch in some artistic style $\mathbf{J}^{\text {rnd }}$.

paper makes the following contributions.

(1) It presents a compact 3-layer hair representation and identifies a dictionary of five types of primitives (eleven types with directions) for the vector field of hair flows (see Fig. 5 and Fig.4). Our hair representation is, in spirit, similar to the primal sketch model[6] for image representation.

(2) It presents a Monte Carlo algorithm for computing the vector fields, especially inferring the directions of the hair by propagating global information in the sketch graph (see Fig.7).

(3) It presents a generative method for hair sketching which is more concise than the example-based discriminative methods for human portrait[4].

The paper is organized as follows. Section 2 presents the three-layer hair model. Section 3 discusses the inference algorithm in two steps: (i) inferring the sketches $\mathbf{S}$ without direction, and (ii) inferring the directions of sketches. Then we show experimental results in Section 4. Some future work is discussed in Section 5.

\section{A generative sketch model of hair}

Our model is illustrated in Fig.2. We first do a Luv transform and decouple a color image $\mathbf{I}^{\text {obs }}$ into a color channel image $\mathbf{I}_{\mathrm{UV}}^{\mathrm{obs}}$ and an intensity image $\mathbf{I}_{\mathrm{Y}}^{\text {obs }}$. The latter is further decomposed into a low frequency band $\mathbf{I}_{\mathrm{L}}^{\text {obs }}$ for global illumination and shading, and a high frequency band $\mathbf{I}_{\mathrm{H}}^{\text {obs }}$ for the hair pattern. We will focus our discussion on $\mathbf{I}_{\mathrm{H}}^{\text {obs }}$ with a three-layer generative model on a lattice $\Lambda$.

The hair pattern $\mathbf{I}_{\mathrm{H}}$ is generated by a hidden layer - the vector field (or flow field) $\mathbf{V}$. The vector field $\mathbf{V}$ is in turn generated by a hair sketch $\mathbf{S}$ which is a number of sketch curves representing the boundaries of hair strands and wisps with direction $\mathbf{d}$.

$$
\text { Sketch }(\mathbf{S}, \mathbf{d}) \stackrel{\Delta_{\text {sk }}}{\longrightarrow} \text { Vector field } \mathbf{V} \longrightarrow \text { hair image } \mathbf{I}_{\mathrm{H}}
$$

$\Delta_{\text {sk }}$ is a dictionary of distinctive primitives for the hair sketch shown in Fig.3 and Fig.4.

In the following, we present the three-layer model in topdown as it is shown in the right side of Fig.2.

\subsection{Layer 1: the hair sketch $S$}

The hair sketches $\mathbf{S}$ consists of a number of curves, and each curve corresponds to a long stroke in the artistic sketch.

$$
\mathbf{S}=\left(N_{C},\left\{C_{i}: i=1,2, \ldots, N_{C}\right\}\right)
$$

These curves are undirected and represent the noticeable structures and discontinuities in the hair flow (or vector field in next subsection). They consist of short line-segments with a patch window called a "primitive". We define 5 types of undirected primitives which make 11 directed primitives for the vector field $\mathbf{V}$, as Fig.3 shows. Each primitive specifies the orientations in $[0, \pi)$ and directions $(-1,+1)$ of the hair flow on the two sides of the line-segment.

(a). Side boundary: the hair flows along one side and the other side is non-hair. By analogy, it is like the bank of a river. See E in Fig.4.

(b). Source and sink: the origin or end of hair flow. See $\mathrm{B}$ and $\mathrm{F}$ in Fig.4. 


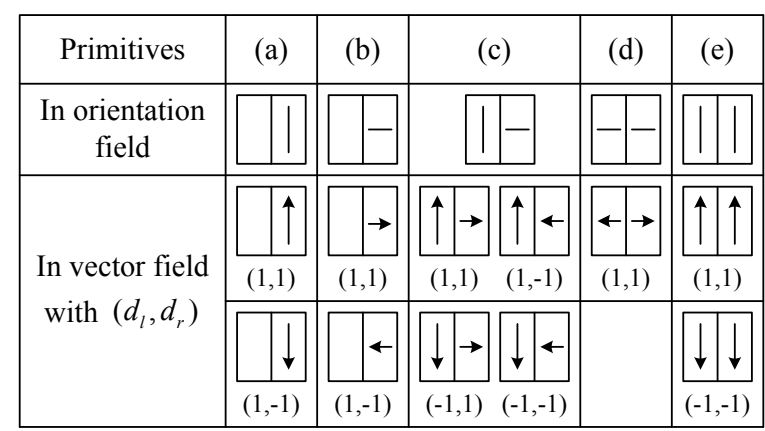

Figure 3. Five primitives of hair orientation field, and eleven primitive in the directed vector field in a dictionary of primitives $\Delta_{\mathrm{sk}}$. (a) Side boundary. (b) Source (origin) or sink (end) of hair strands. (c) Occluding boundary. (d) Dividing line. (e) Stream line.

(c). Occluding boundary: the boundary of two hair strands of different orientations. See A in Fig.4.

(d). Dividing line: hair growth in same orientation but reverse directions, it often occurs at the top the hair. See D in Fig.4.

(e). Stream line: the hair flow with strong contrast. See C in Fig.4.

The primitives are associated with a number of variables (or parameters) which are denoted by

$$
\mathbf{B}=\left(\ell, x, y, \theta, \rho,\left(\theta_{l}, \theta_{r}\right)\right)
$$

,where $\ell \in\{a, b, c, d, e\}$ is the type, $(x, y)$ is the position, $\theta$ is its orientation in $[0, \pi)$, and $\left(\theta_{l}, \theta_{r}\right)$ are the orientation angles at the left and right side flows and thus they allow some variations from the orientations defined in the primitive templates.

The directions are important for hair understanding and stylistic rendering, and their inference needs global information and thus is studied separately from the orientation.

Each primitive has a direction $d=\left(d_{l}, d_{r}\right)$ specifying the flow direction at the two sides. We request all primitives in a curve have the same type $\ell$ and same directions $d=$ $\left(d_{l}, d_{r}\right)$, therefore we write the directed curve as,

$$
(C, \mathbf{d})=\left(\ell, N_{\mathbf{B}},\left\{\mathbf{B}_{j}: j=1,2, \ldots, N_{\mathbf{B}}\right\},\left(d_{l}, d_{r}\right)\right) .
$$

The role of the sketch layer $\mathbf{S}$ is to ensure global compatibility by introducing prior knowledge necessary for inferring the vector field and artistic rendering. The prior model for $\mathbf{S}$ is product of

$$
p(\mathbf{S}, \mathbf{d})=p\left(N_{C}\right) \prod_{C_{i} \in \mathbf{S}} p\left(C_{i}\right) p\left(\mathbf{d}_{i}\right) \prod_{<C_{i}, C_{j}>} p\left(\mathbf{d}_{i}, \mathbf{d}_{j}\right) .
$$

The above $p(\mathbf{S}, \mathbf{d})$ consists of the following terms,

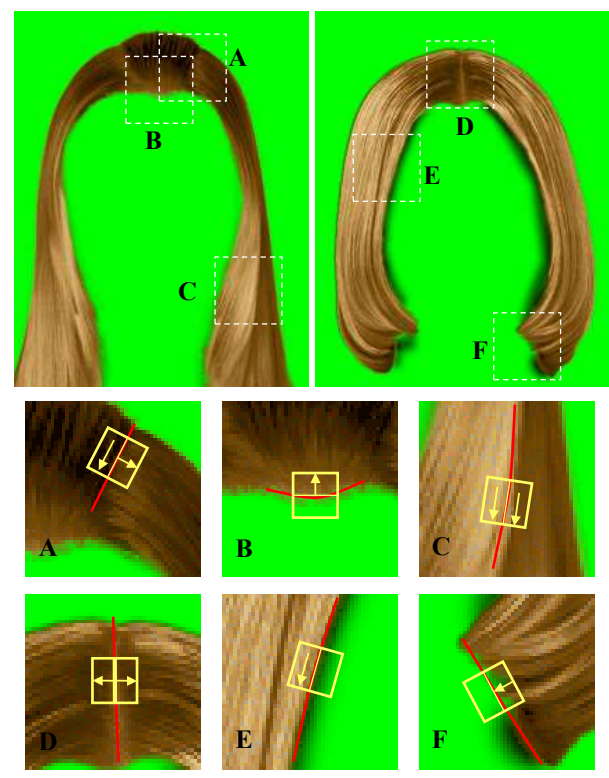

Figure 4. Examples of primitives of hair sketch.

(i) $p\left(N_{c}\right) \propto \exp ^{-\lambda_{o} N_{C}}$ penalizes the number of curves.

(ii) $p(C)$ is the prior for each curve without direction information.

$$
p(C)=\prod_{\mathbf{B}_{i} \in C} p\left(\rho_{i}\right) \prod_{<\mathbf{B}_{i}, \mathbf{B}_{j}>} p\left(\theta_{i}, \theta_{j}\right)
$$

, where the smoothness in orientation of two consecutive primitives $<\mathbf{B}_{i}, \mathbf{B}_{j}>$ is modelled by a Gaussian distribution $p\left(\theta_{i}, \theta_{j}\right)=N\left(\theta_{i}-\theta_{j} ; 0, \sigma_{s}\right)$.

And strong gradients $\rho_{i}$ for each primitive $\mathbf{B}_{i} \in C$ is modelled by $p\left(\rho_{i}\right)$. Due the the heavy tail of $p\left(\rho_{i}\right)$, we fit a mixture Gaussian distribution with two kernels. $p\left(\rho_{i}\right)$ is shown in Fig.5(b) which is learned from manually labeled sketches and vector fields (see experiment).

(iii) The direction $\mathbf{d}_{i}$ for each curve $C_{i} \in \mathbf{S}$ is biased by a pointwise prior $p\left(x_{i}, y_{i}, \theta_{i}, d_{i}\right)$ which means how likely the flow vector takes a certain orientation $\theta \in[0, \pi)$ and direction $d \in\{-1,+1\}$. This prior is shown in Fig.5(a) and is learned from training images using manually labeled sketches. Thus, the probability of the direction of a curve is defined as,

$$
\left.p\left(\mathbf{d}_{i}\right)\right) \propto \prod_{v \in C_{i}} p\left(v_{x}, v_{y}, \theta(v), d\right)
$$

(iv) $p\left(\mathbf{d}_{i}, \mathbf{d}_{j}\right)$ is the compatibility of directions of two adjacent curves $C_{i}, C_{j}$. (The details will be explained in Section 3.2 and shown in Fig. 7.)

\subsection{Layer 2: the vector field V}

The vector field $\mathbf{V}=(\boldsymbol{\Theta}, \boldsymbol{\rho}, \mathbf{d})$ includes three components. 


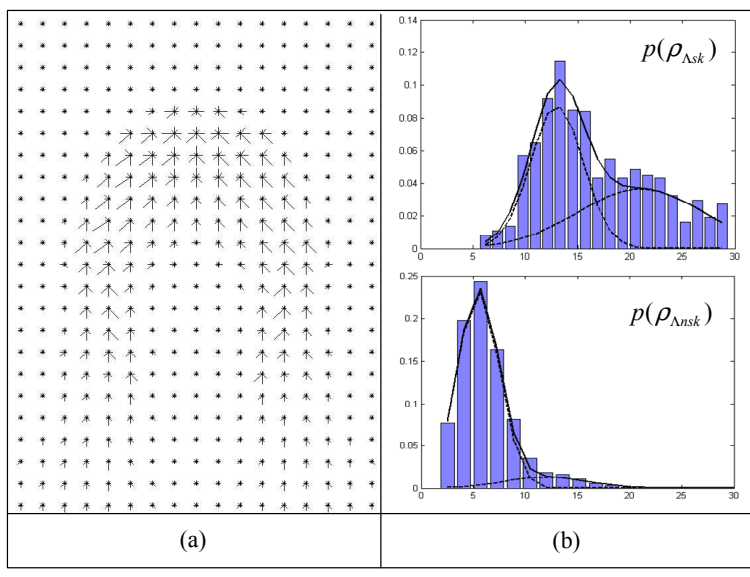

Figure 5. (a) The point-wise prior probability $p(x, y, \theta, d)$ for the flow vector $(\theta, d)$ at $(x, y)$. The long arrow means high probability. (b) The prior probability $p(\rho)$ for the intensity gradient perpendicular to the orientation $\theta$ in sketchable part and non-sketchable part.

1. An orientation field for the local hair orientation in $[0, \pi)$.

$$
\boldsymbol{\Theta}=\{\theta(v): \theta(v) \in[0, \pi), v \in \Lambda\}
$$

2. A gradient strength field for the intensity contrast,

$$
\boldsymbol{\rho}=\left\{\rho(v): \rho(v)=\nabla_{\perp \theta(v)} \mathbf{I}_{\mathrm{H}}, v \in \Lambda\right\}
$$

3. A direction field $\mathbf{d}$ (upwards or downwards) respectively at each pixel.

$$
\mathbf{d}=\{d(v): d(v) \in\{-1,+1\}, v \in \Lambda\} .
$$

At each point $v$, the orientation plus direction yields the flow vector $\tau(v) \in[0,2 \pi]$.

The sketch $\mathbf{S}$ divides the lattice $\Lambda$ into two disjoint areas $\Lambda_{\mathrm{sk}}$ and $\Lambda_{\mathrm{nsk}} . \Lambda_{\mathrm{sk}}$ are covered by the rectangular windows of the primitives. And the vector field copies the flow from the primitive at each window as in Fig. 6(c).

The remaining lattice $\Lambda_{\text {nsk }}$ corresponds to areas with usually flat and noisy intensity, and its vector flow is "filledin" from $\Lambda_{\text {sk }}$ by a smoothness probability $p\left(\mathbf{V}_{\Lambda_{\text {nsk }}} \mid \mathbf{V}_{\Lambda_{\text {sk }}}\right)$ on the orientation and direction $\mathbf{V}$ which measures the distance of adjacent flows. We combine the direction information into orientation and thus have $(\theta, d) \rightarrow \tau$ with $\tau \in[0,2 \pi]$. Thus,

$$
p\left(V_{\Lambda n s k} \mid V_{\Lambda s k}\right) \propto \prod_{v \in \Lambda n s k} p\left(\rho_{v}\right) \cdot \exp \left\{-\sum_{v \in \Lambda n s k} \sum_{u \in \partial v} e\left(\tau_{v}, \tau_{u}\right)^{2} / \lambda_{\theta}\right\}
$$

,where $\partial v$ is the 4-nearest-neighbor of $v$ and $p\left(\rho_{v}\right)$ is a prior distribution for $\rho$ in $\Lambda_{\text {nsk }}$ as shown in Fig. 5. The distance $\mathrm{e}\left(\tau_{v}, \tau_{u}\right)$ is defined on a circle $[0,2 \pi]-\mathrm{a}$ Riemannian space. Thus we adopt the following distance[15, 14].

$$
\operatorname{dist}\left(\tau_{v}, \tau_{u}\right)=\sqrt{2\left(1-\cos \left(\tau_{v}-\tau_{u}\right)\right.},
$$

To find the optimal solution of equation(2), we can use gradient decent with the diffusion equation

$$
\frac{d \tau_{v}}{d t}=\sum_{u \in \partial v} \sin \left(\tau_{v}-\tau_{u}\right)
$$

See the computed vector field $\mathbf{V}^{\text {syn }}$ in Fig. 6(d). The gradient strength $\rho_{v}$ is computed from the image in bottomup step.

\subsection{Layer 3: the image layer $I_{H}$}

The image layer $\mathbf{I}_{\mathrm{H}}$ is controlled by the vector field, very much like the LLC visualization of the vector field[2]. Intuitively, we need to generate an image so that it has low intensity changes along the flow direction $\theta(v), v \in \Lambda$ for the hair, while it keeps some intensity contrast between hairs according to $\rho(v)$ perpendicular to $\theta_{v}$.

$p\left(\mathbf{I}_{\mathrm{H}} \mid V\right) \propto \exp \left\{-\sum_{v \in \Lambda} \frac{\left(\nabla_{\theta(v)} \mathbf{I}_{\mathrm{H}}\right)^{2}}{2 \sigma_{\theta}^{2}}+\frac{\left(\left\|\nabla_{\perp \theta(v)} \mathbf{I}_{\mathrm{H}}\right\|-\rho(v)\right)^{2}}{2 \sigma_{\rho}^{2}}\right\}$

The synthesized $\mathbf{I}_{\mathrm{H}}$ is sampled from the above model. Then, one can easily reconstruct a color image by adding the shading and color bands. See Fig.2 (right side).

Summarizing the three-layer model, we obtain the generative sketch model of hair,

$$
p\left(\mathbf{I}_{\mathrm{H}}, \mathbf{V},(\mathbf{S}, \mathbf{d})\right)=p\left(\mathbf{I}_{\mathrm{H}} \mid \mathbf{V}\right) p\left(\mathbf{V} \mid(\mathbf{S}, \mathbf{d}) ; \Delta_{\mathrm{sk}}\right) p(\mathbf{S}, \mathbf{d})
$$

\section{Infer the sketch and vector field}

Given an input image $\mathbf{I}^{\text {obs }}$, we infer the vector field $\mathbf{V}=(\boldsymbol{\Theta}, \boldsymbol{\rho}, \mathbf{d})$ and the directed sketch $(\mathbf{S}, \mathbf{d})$ by maximizing the Bayesian posterior. As the gradient strength $\rho(v)$ is the image gradient at orientation $\theta(v)$, and is deterministic.

Also the direction of the vector field is determined by the direction in the sketch. With a bit abuse of notation, we use $\mathbf{d}$ to represent all the directions, thus our objective is to optimize,

$$
(\boldsymbol{\Theta}, \mathbf{S}, \mathbf{d})^{*}=\arg \max p\left(\mathbf{I}_{\mathrm{H}}^{\mathrm{obs}} \mid \mathbf{V}\right) p\left(\mathbf{V} \mid \mathbf{S} ; \Delta_{\mathrm{sk}}\right) p(\mathbf{S}, \mathbf{d}) .
$$

\subsection{The greedy algorithm}

The global optimization of the above posterior is computationally intensive. We take the following greedy algorithm which augment one primitive at each step like matching pursuit[10]. Given $\mathbf{I}_{\mathrm{H}}^{\mathrm{obs}}$,

1. Initialize $\mathbf{S}=\emptyset, \Lambda_{\mathrm{sk}}=\emptyset, \Lambda_{\mathrm{nsk}}=\Lambda, \mathbf{d}=$ nil.

2. Initialize $\boldsymbol{\Theta}=\arg \max p\left(\mathbf{I}_{\mathrm{H}}^{\mathrm{obs}} \mid \boldsymbol{\Theta}\right) p(\boldsymbol{\Theta})$. 


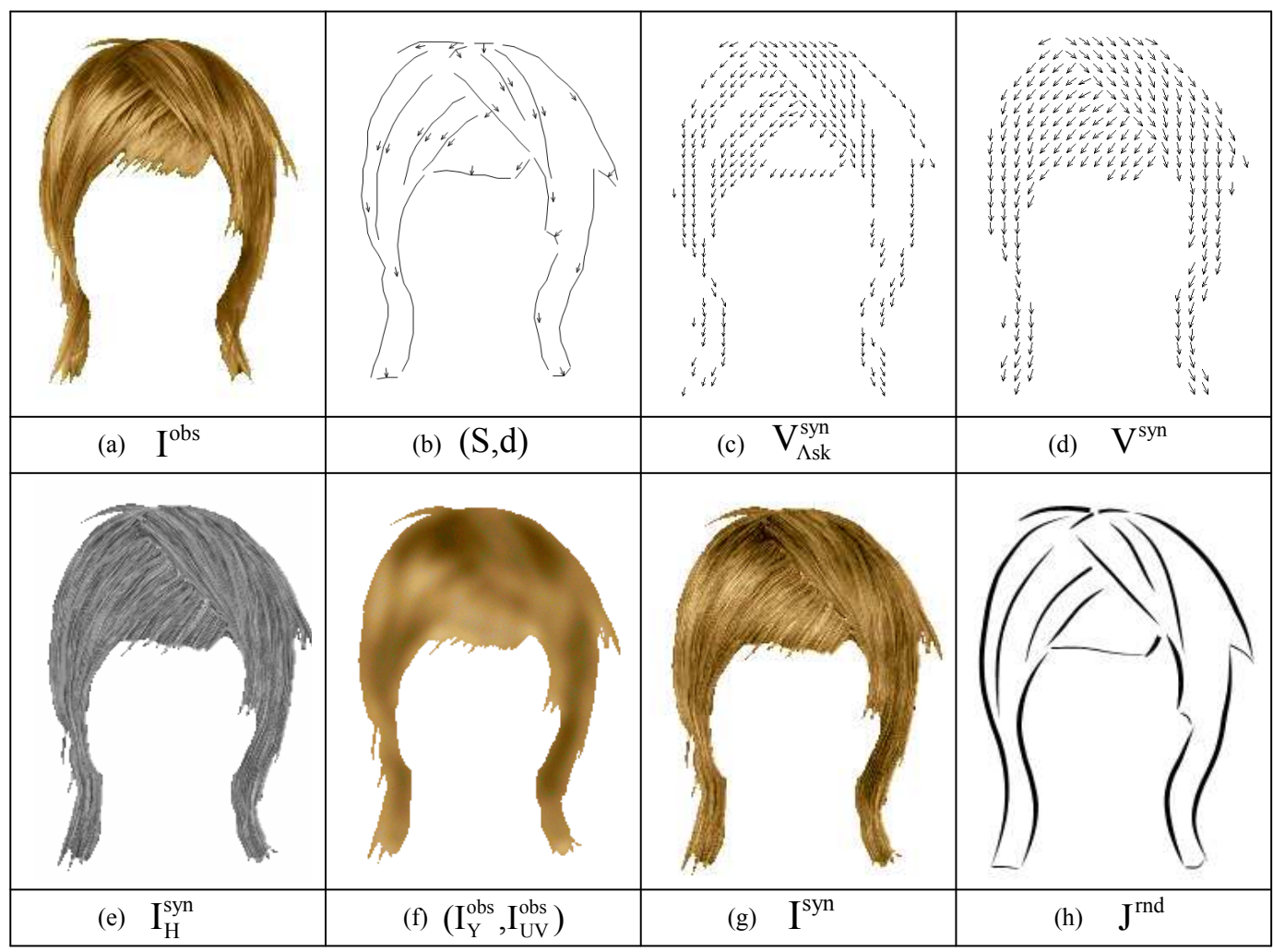

Figure 6. Example of hair model and inference. For input image $\mathrm{I}^{\mathrm{obs}}$ in (a), the sketch $\mathrm{S}$ with directions is computed in (b) which generate the vector field in sketch part $\mathrm{V}_{\Lambda_{\mathrm{sk}}}$ in (C). With $V_{\Lambda_{\mathrm{sk}}}$ being boundary condition, we fill in the vector field in non-sketch part to get the full vector field $V$ in (d). The vector field $\mathrm{V}$ in turn synthesizes the high frequency part of hair $\mathrm{I}_{\mathrm{H}}^{\mathrm{syn}}$ in (e). After adding the shading and color bands $\left(I_{Y}^{\text {obs }}, I_{U V}^{\text {obs }}\right)$ in (f), we get the synthesis result $I^{\text {syn }}$ in $(g)$. At the same time, we render an artistic sketch $\mathbf{J}^{\text {rnd }}$ in (h).

\section{Iterate steps 4-8 (like matching pursuit)}

4. $\quad$ Compute a set of candidate sketch primitives from the non-sketch orientation field $\Theta_{\Lambda_{\text {nsk }}}$ with weights,

$$
\left\{\left(\mathbf{B}_{i}^{+}, \omega_{i}^{+}\right): i=1,2, \ldots, M\right\} .
$$

5. Find the best $\mathbf{B}^{*}$ with highest weight $\omega^{*}$.

6. If $\omega^{*}<\epsilon$, goto step 9 , otherwise

7. $\quad$ Add $\mathbf{B}^{*}$ and its window $\Lambda\left(\mathbf{B}^{*}\right)$ into the sketch part.

8. Update $\boldsymbol{\Theta}=\arg \max p\left(\mathbf{I}_{\mathrm{H}}^{\mathrm{obs}} \mid \boldsymbol{\Theta}\right) p(\boldsymbol{\Theta} \mid \mathbf{S})$

9. Compute the direction of $\mathbf{d}$ for curves and orientation field.

We briefly mention a few details in the above steps. In step 2 and step 8 , the probability for updating $\Theta$ is

$$
p\left(\mathbf{I}_{\mathrm{H}}^{\mathrm{obs}} \mid \boldsymbol{\Theta}\right) p(\boldsymbol{\Theta} \mid \mathbf{S}) \propto p\left(\boldsymbol{\Theta}_{\Lambda_{\mathrm{nsk}}} \mid \boldsymbol{\Theta}_{\Lambda_{\mathrm{sk}}}\right) \exp \left\{-\sum_{v \in \Lambda} \frac{\left(\nabla_{\theta(v)} \mathbf{I}_{\mathrm{H}}^{\mathrm{obs}}\right)^{2}}{2 \sigma_{\theta}}\right\}
$$

, where the second part is the likelihood probability that the intensity gradient along the tangent angle (hair) $\theta(v)$ should be small everywhere. It comes from eqn. (5). The first factor $p\left(\boldsymbol{\Theta}_{\Lambda_{\text {nsk }}} \mid \boldsymbol{\Theta}_{\Lambda_{\text {sk }}}\right)$ is a smoothness constraint similar to eqn. (2) while $\theta$ is $\pi$ periodic. We solve its diffusion equation by Gaussian-Seidel method as in [15, 14].

In step 4 , the candidate set is computed by fitting the primitives in the dictionary $\Delta_{\mathrm{sk}}$ at various locations, orientations, and scales to the current orientation field $\Theta_{\Lambda_{\text {nsk }}}$ (the non-sketch part). To save computation, we may only need to compute at the "promising" pixels which have some salient intensity gradients and orientation discontinuities, using some filters similar to the primitives.

The weight $\omega_{i}^{+}$for a primitive $\mathbf{B}_{i}^{+}$is a probability measure coming from three components.

1. The likelihood probability or fitness at the window $\Lambda\left(\mathbf{B}_{i}^{+}\right) \cdot p\left(\boldsymbol{\Theta}_{\Lambda\left(\mathbf{B}_{i}^{+}\right)} \mid \mathbf{B}_{i}^{+}\right)$.

2. Suppose there is an existing curves $C$ in the current sketch $\mathbf{S}$ and $C$ is close to $\mathbf{B}_{i}^{+}$. The prior probability from the smoothness of curves $p(C)$ will favor the primitives that have good continuity in space and orientation. The the sec- 


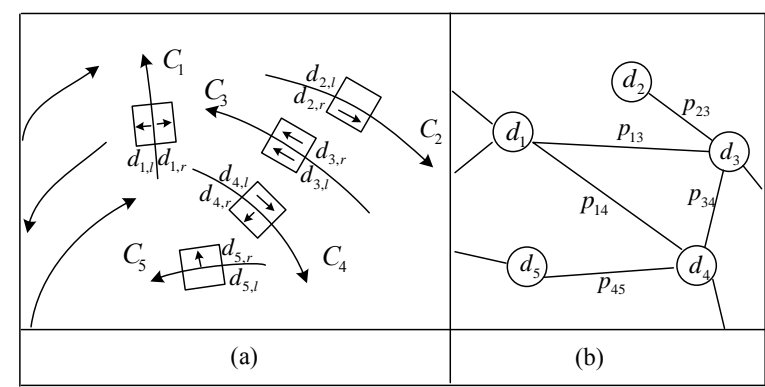

Figure 7. (a) A portion of a sketch with a number of curves. (b) An adjacency graph where each curve in (a) is represented by a node. Two adjacent nodes (curves) have a weighted edge for their direction compatibility constraints. $p\left(\mathbf{d}_{i}, \mathbf{d}_{j}\right)$ is abbreviated by $p_{i j}$.

ond term is a conditional probability $p\left(\mathbf{B}_{i}^{+} \mid C\right)$ derived from eqn.(1).

3. Also we need to compare with the non-sketchable probability or fitness $p\left(\boldsymbol{\Theta}_{\Lambda_{\text {nsk }}} \mid \boldsymbol{\Theta}_{\Lambda_{\text {sk }}}\right)$ at the window $\Lambda\left(\mathbf{B}_{i}^{+}\right)$. We denote it as $p\left(\boldsymbol{\Theta}_{\Lambda\left(\mathbf{B}_{i}^{+}\right)}\right)$.

Therefore we have

$\omega_{i}^{+}=\log p\left(\boldsymbol{\Theta}_{\Lambda\left(\mathbf{B}_{i}^{+}\right)} \mid \mathbf{B}_{i}^{+}\right)+\log p\left(\mathbf{B}_{i}^{+} \mid C\right)-\log p\left(\boldsymbol{\Theta}_{\Lambda\left(\mathbf{B}_{i}^{+}\right)}\right)$.

In step 8, we do not have to update $\Theta$ every time after we add a new sketch primitive. To save computation, we may only do it once we pursuit a whole curve.

\subsection{Infer the direction of sketch}

Suppose we have a set of undirected sketch curves $\left\{C_{1}, \ldots, C_{N_{C}}\right\}$ computed from steps 1-8 in the above algorithm. We need to assign correct directions to the two sides of each curves.

In this step, each curve $C_{i}$ need to assign a direction $\mathbf{d}_{i}=\left(d_{l, i}, d_{r, i}\right) . \mathbf{d}_{i}$ has $2,2,4,1,2$ possible choices for the five types of undirected primitives in Fig. 3(a)-(e) respectively. Let's denote $\mathbf{d}=\left\{\mathbf{d}_{i}: i=1, . ., N_{C}\right\}$ as the direction of $\mathbf{S}$.

As we mentioned, we have the direction prior $p\left(\mathbf{d}_{i}\right)$ for each curve $C_{i}$ in (2.1).

A naive direction inference method is to assign the direction of each curve just by the prior. However, lacking of compatibility information between curves, it fails for a lot of cases, such as in Fig. 8(g).

To use the compatibility information, we define a adjacency graph with the $N_{C}$ curves $\left\{C_{1}, \ldots, C_{N_{C}}\right\}$ being the vertices. And two adjacent curves have a connection in the graph weighted by directional compatibility $p\left(\mathbf{d}_{i}, \mathbf{d}_{j}\right)$. For curve $C_{i}$ and $C_{j}$, we denote the local neighbor region by $\Lambda_{N\left(C_{i}, C_{j}\right)}$. Then, for different configurations of $\left(\mathbf{d}_{i}, \mathbf{d}_{j}\right)$, we define the directional compatibility as

$$
p\left(\mathbf{d}_{i}, \mathbf{d}_{j}\right) \propto \max _{V_{\Lambda_{N}\left(C_{i}, C_{j}\right)}} p\left(V_{\Lambda_{N\left(C_{i}, C_{j}\right)}} \mid C_{i}, C_{j}, \mathbf{d}_{i}, \mathbf{d}_{j}\right)
$$

, where $p\left(V_{\Lambda_{N\left(C_{i}, C_{j}\right)}} \mid C_{i}, C_{j}, \mathbf{d}\left(C_{i}\right), \mathbf{d}\left(C_{j}\right)\right)$ is defined by eqn.(2).

Given the adjacency graph, the direction inference becomes a typical graph labeling problem,

$$
\mathbf{d}^{*}=\arg \max \prod_{C_{i}} p\left(\mathbf{d}_{i}\right) \prod_{<C_{i}, C_{j}>} p\left(\mathbf{d}_{i}, \mathbf{d}_{j}\right) .
$$

We adopt a graph labeling algorithm called the Swendson-Wang cut which can rapidly find a global solution for $N_{c}=10 \sim 30$ through Markov chain Monte Carlo search. Once the curves are directed in step 9, propagating the direction to the orientation field is straightforward using the smoothness prior for $\mathbf{V}_{\text {nsk }}$ in eqn.(2).

\section{Experiments}

We collected a hair dataset containing 300 human hair images extracted from a cosmetic makeup software. The hairs are pasted on a background with constant color, we segmented the hair out using the background color as the color key.

We labelled the sketch of 20 hairs with distinct style as the training examples. From the training data, we get the ideal vector field for each training example through the diffusion eqn. (4). We learn the two prior probabilities used in the representation. $p(\theta, d)$ and $p(\rho)$ shown in Fig.5. We also learned a number of parameters used in the representation.

We illustrate the stepwise results of the algorithm in Fig. 6 and more results in Fig. 9.

Our experiments reveal two important contributions of the sketch layer $\mathbf{S}$ in computing the vector field.

1. As shown in Fig. 8(a)-(e), the knowledge of primitives corrects errors in the initial orientation field $(\rho, \theta)$, especially around the discontinuities of flows where the resolution may be low because we need to pool information in a local area.

2. It helps to determine the directions which cannot be determined locally. As in Fig. 8(f)-(j), the compatibility between curves plays an important role in direction inference algorithm.

With the sketch, we have the high level information of hair structure, such as the dividing line, the source and sink boundary. For each curve in the sketch, we render it by a smoothing stroke in a particular style with the width determined by its type, direction and strength. We use such a rendering procedure to get the rendering result $\mathbf{J}^{r n d}$. 


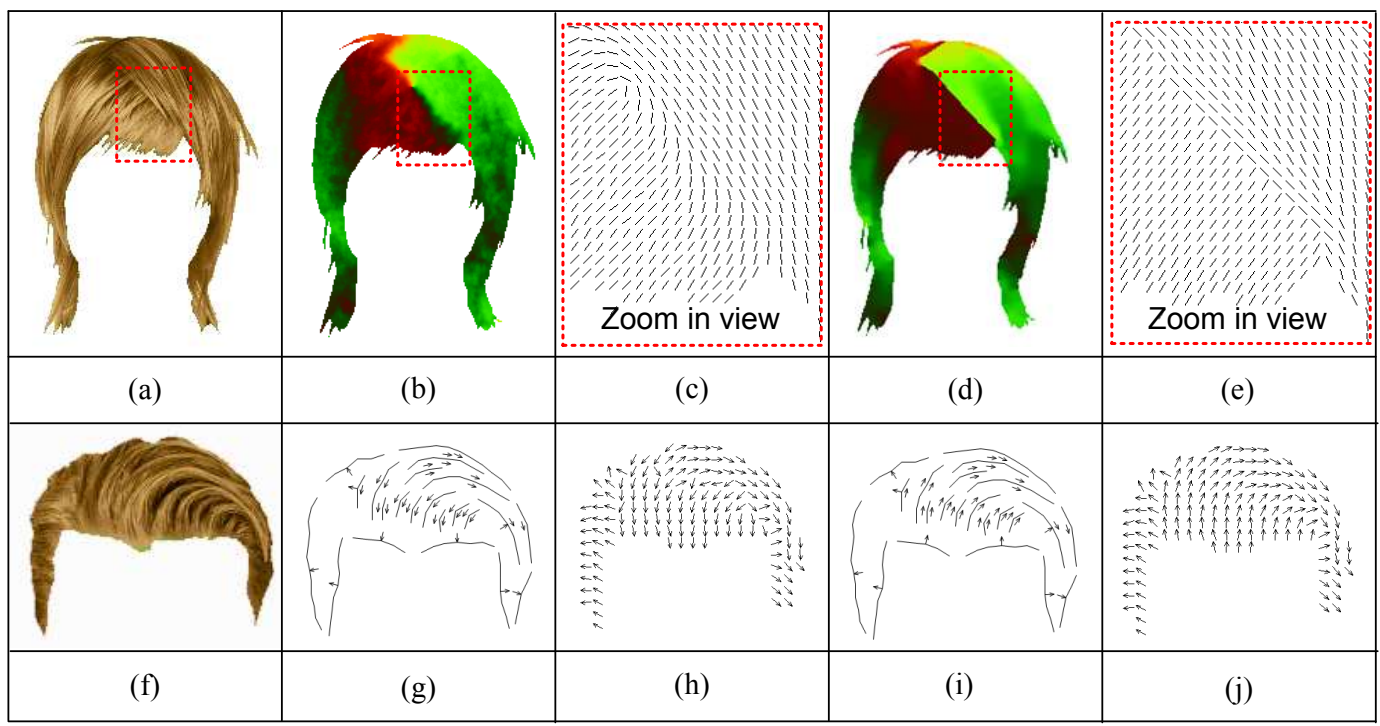

Figure 8. (a)-(e) Comparison of orientation field inference with/out sketch. (f)-(j) Comparison of direction inference with/out curve compatibility. (a) original image; (b)(d) orientation field inferred without/with sketch; (c)(e) zoom in views of orientation field; (f) original image; (g) sketch with direction inferred by point wise prior; (h) vector field generated by (h); (i) sketch with direction inferred by point wise prior with curve compatibility; (j) the vector field generated by (i);

\section{Future work}

We are currently encoding more knowledge about hair structures in our model, so that our method can handle more complex hair styles including pigtail, braid, and curly hairs. We should also improve the rendering style with more controllability.

\section{Acknowledgement}

This work is supported by grants NIH 5R01-EY013875, NSF IIS-0222967, and a Microsoft gift. The authors thank Dr. Heung Yeung Shum and Dr. Ying Qing Xu for many stimulating discussions.

\section{References}

[1] J.R.Bergen and E.H.Adelson, "Theories of visual texture perception," Spatial Vision D.Regan (eds.)CRC Press, 1991.

[2] B. Cabral and L.C. Leedom, "Imaging vector fields using integral convolution," Proc. 20th Conf. on Comp. Graphics and Interactive Techniques, pp 263-270, ACM Press, 1993.

[3] J.T. Chang, J.Y. Jin, and Y.Z. Yu, ”A practical model for hair mutual interactions," Proc. of Siggraph/Eurographics Symp. on Computer Animation, 2002.

[4] H. Chen and Z.Q. Liu, C. Rose, Y.Q. Xu, H.Y. Shum and D. Salesin, "Example-based composite sketching of human portraits", Proc. 3rd Int'l Symp. NPAR, 95-153, 2004.
[5] P.Pérez, A.Blake and M.Gangnet, "JetStream: probabilistic contour extraction with particles", ICCV, 524-531, 2001.

[6] C.E. Guo, S.C. Zhu, and Y.N. Wu, "A mathematical theory for primal sketch and sketchability," Proc. of ICCV, 2003.

[7] M Kass and A Witkin, "Analyzing oriented patterns," CVGIP, 37, 362-385, 1987.

[8] T.Y. Kim and U. Neumann, "Interactive multi-resolution hair modeling and editing," Proc. of Siggraph, 2002.

[9] D.W Lee and H. S. Ko, "Natural hairstyle modeling and animation," Graphical Models, 63, 67-85, 2001.

[10] S. Mallat and Z. Zhang, "Matching pursuit in a timefrequency dictionary," IEEE Trans. on Signal Proc. 41. 33973415, 1993.

[11] M. Nitzberg, D.B. Mumford, and T. Shiota, "Filtering, segmentation, and depth," Lecture Notes in CS, Springer-Verlag, Vol 662, 1993.

[12] S.W. Zucker, "Early orientation selection: tangent fields and the dimensionality of their support," CVGIP, 32, 74-103, 1985.

[13] A. Barbu and S.C. Zhu, "Graph partition by Swendsen-Wang cuts", Proc. of ICCV, 320-327, 2003.

[14] T. Chan and J.H. Shen, "Variational restoration of nonflat image features: models and algorithms", SIAM J. on Applied Math, Vol 61, 1338-61, 2001.

[15] P. Perona, "Orientation diffusions", IEEE Trans. on Image Proc., 100-107, 1999.

[16] S.Paris, H.M.Briceno and F.X.Sillion, "Caputer of hair geometry from multiple images", Proc. of Siggraph, 2004. 


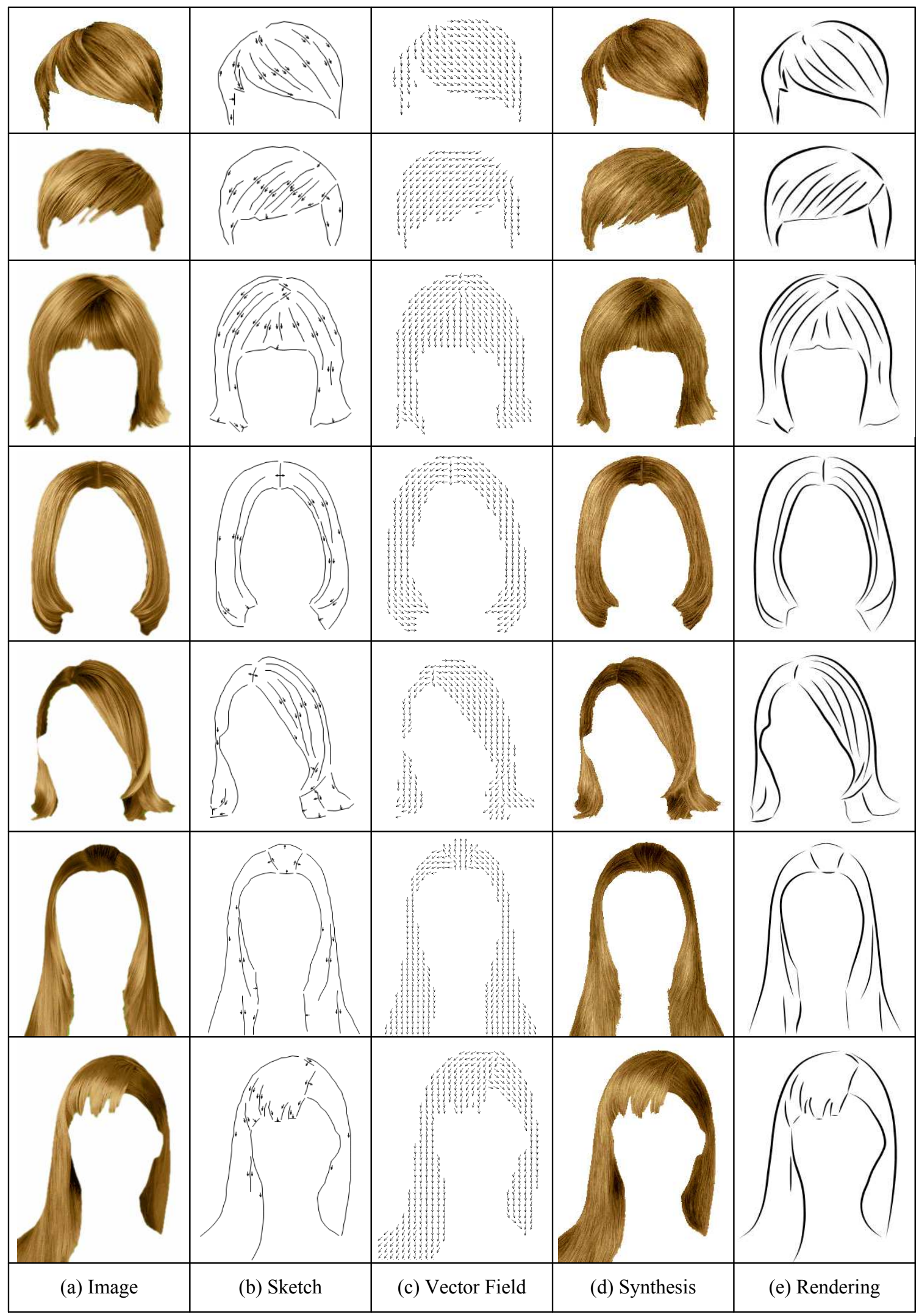

Figure 9. More results of our algorithm. 